\title{
Temperature Measurement in a TEM using Electron Diffraction of Amorphous Films
}

\author{
Misa Hayashida ${ }^{1}$, Kai Cui ${ }^{1}$, Marek Malac ${ }^{1,2}$
}

\author{
1. National Institute for Nanotechnology 11421 Saskatchewan Drive, Edmonton, T6G 2M9, Canada \\ ${ }^{2}$ Department of Physics, University of Alberta, Edmonton, T6G 2E1, Canada
}

Several methods for measuring temperature of a TEM sample by electron diffraction have been reported [1,2]. Crystalline heavy metal nanoparticles have been used because their diffraction rings are sharp and have sufficient intensity to measure the radii that decreases with increasing temperature. The use of heavy metal nanoparticles complicates sample preparation. Furthermore, it can lead to contamination of the studied sample by the heavy metal of the nanoparticles deposited on the sample to measure temperature. Instead, we used standard amorphous $30 \mathrm{~nm}$ SiN. The amorphous SiN exhibits broad diffraction rings, but are used as TEM sample support films. Therefore the use of amorphous SiN does not require additional sample preparation step and does not introduce potential sample contamination.

The measurements have been performed in a HITACHI H-9500 environmental transmission electron microscope (ETEM) operated at $300 \mathrm{kV}$ acceleration voltage. The diffraction patterns were collected with a camera length $\mathrm{L}=0.5 \mathrm{~m}$ using a Gatan Orius ${ }^{\mathrm{TM}} \mathrm{CCD}$ camera binned by a factor of 2 to $1336 \times 2004$ pixels. Eight subsequent diffraction patterns with 5 s exposure time were acquired at each temperature. Figure 1 shows a) the as collected diffraction patterns and b) diffraction pattern after contrast enhancement.

To locate the center position of the patterns, we used binary (black and white) template images shown in Figure 1c. The cross-correlation between a template image c) and a contrast enhanced image b) was calculated for various diameter and width of the ring in the template image c). The location of the template image and its position with the highest cross correlation coefficient in the cross correlation peak position was used as center of the diffraction pattern. An averaged radial profile of the DP in a) was calculated using the above DP centre.

Figure 2 shows the temperature dependence of radii of the amorphous SiN diffraction rings. The distance from diffraction pattern centre to the pixel with maximum intensity of the rings in the radial profile is plotted here. The radius is linearly and inversely related to temperature, as in crystalline materials, as expected from expansion of the material with increased temperature $[1,2]$. When sum of 8 patterns was used (red circles), the linearity was better than when individual images were used (purple dots). The improved linearity arises from increased signal to noise ratio for the summed DP as opposed to individual DPs.

We show that the broad diffraction rings of a standard SiN TEM support film can be fitted with sufficient precision to allow for temperature measurement. The use of standard support film implies that our method and does not require special sample preparation or addition of nanoparticles and can be used to many sample types.

[1] Jonathan P. Winterstein et al., Ultramicroscopy Microsc. Microanal. 21, 1622-1628, 2015.

[2] Florian Niekiel et al., Ultramicroscopy (in press) 
(a)

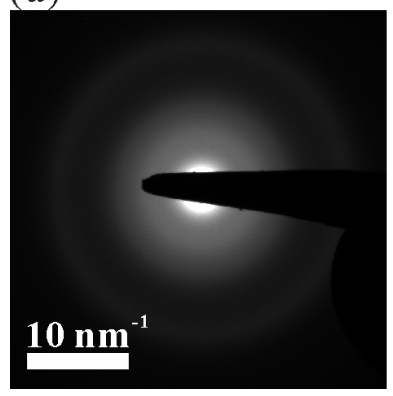

(b)

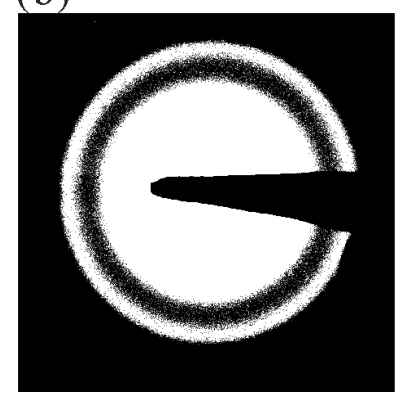

(c)

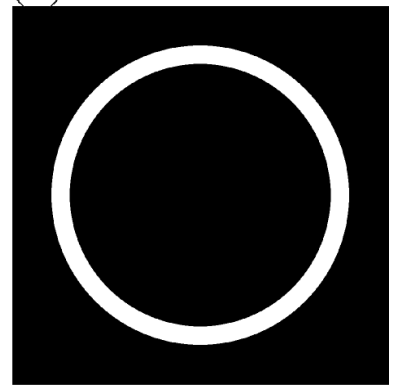

Figure 1. (a) A diffraction pattern from a SiN film. (b) Contrast enhanced image of (a). (c) An example of a template image for center position detection

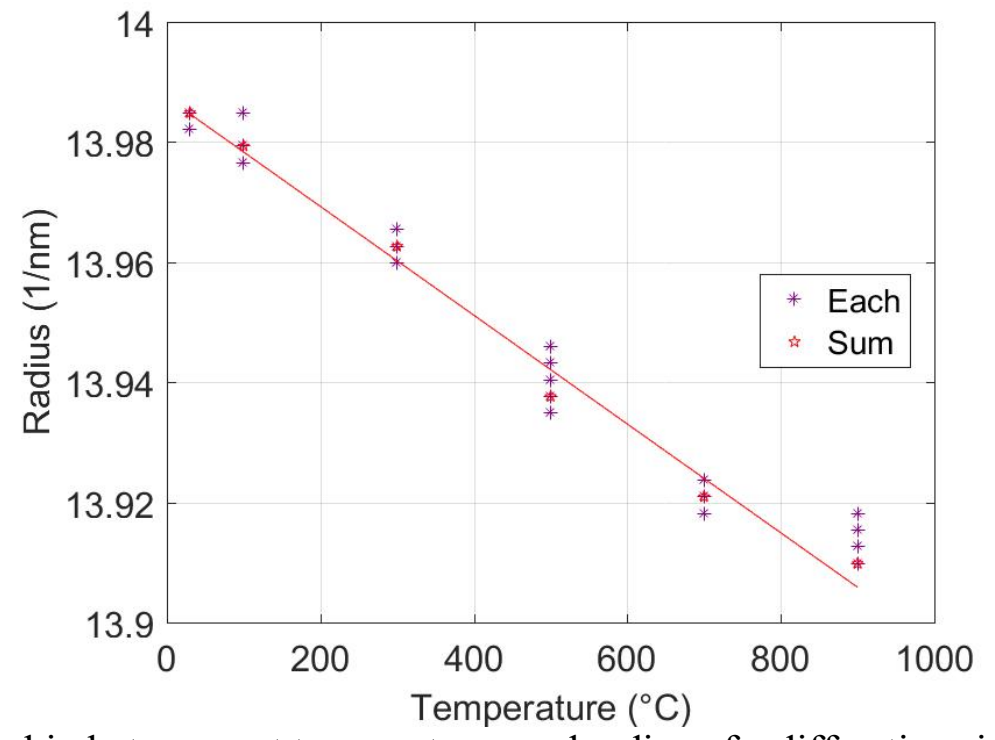

Figure 2. Relationship between set temperatures and radius of a diffraction ring from a SiN film. 